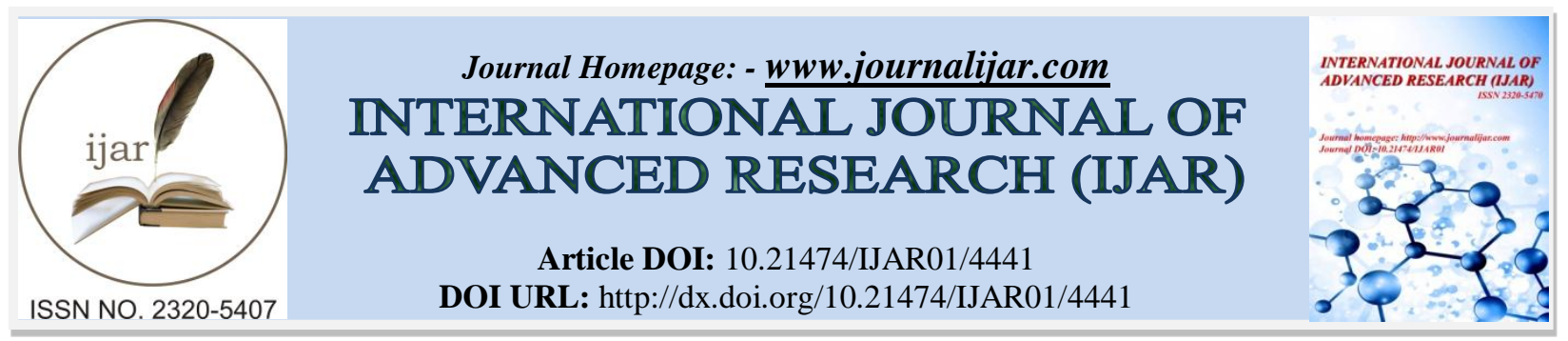

RESEARCH ARTICLE

\title{
MODELING AND SIMULATION OF ELECTROCHEMICAL SUPERCAPACITOR FOR HIGH POWER DELIVERY.
}

Thoudam Paraskumar Singh and Sudhir Y Kumar.

CET, Mody University, Lakshmangarh, Sikar 332311, India.

\section{Manuscript Info}

Manuscript History

Received: 10 April 2017

Final Accepted: 12 May 2017

Published: June 2017

Key words:-

Electrochemical Capacitors; Supercapacitor;

Ultracapacitor; Energy Storage

\section{Abstract}

An Electrochemical Supercapacitor is an energy storage device that can store high energy and also release high power from the same time. Almost all the applications in the field of power transmission, electric or hybrid vehicles, industrial electronics to name some; where battery was used as power source can be replaced by Supercapacitor. This paper first discusses different types of models commonly used and chosen the model which includes the non-linear behavior. OrCAD Lite demo version was used to simulate and obtain the behavior of Supercapacitor by varying the Equivalent Series Resistance. This analysis has the advantage that identify performance of high power release is dependent on the value of Equivalent Series Resistance. With proper selection of electrolyte in fabrication also the power release can be very high which can supply high peak load.

Copy Right, IJAR, 2017,. All rights reserved.

\section{Introduction:-}

The energy in this world can neither be created nor destroyed but it is converted from one form to another. All the energy generated from different types of power plants using renewable and non-renewable resources are utilized as generated. They can be temporarily stored in certain energy storage which is limited in storage capacity. The complete generated energy from power plants can never be stored in one place or no single storage device or any number of combined storage devises together can do the storing. It is only about some fractions in percentage of the total generated. There is growing demand for developing efficient storage system in the field of electric and hybrid vehicles, consumer electronics, renewable energy systems like solar and wind, generating plants and electric energy distribution grid with varying performance characteristics.

The energy storage devices available as of today are not ideal to meet all the economic and technical requirement of the growing demand. Some of the storage devices which see competition amongst the devices are electrochemical batteries; pump hydro, flywheels, Superconducting Magnetic Energy Storage, compressed air energy storage, Fuel Cells etc. The interest in developing and refining more efficient energy storage devices has led to the introduction of Supercacitor and has matures over the decade and emerged with major potential to facilitate advances in storing of energy. The Ragone plot for different energy storage devices is represented to compare the specific power and specific energy in figure $1[3]$.

\section{Working Principle to Store Energy:-}

The most promising energy storage device which can replace battery in many application of present time is the Supercapacitor (SC), also known by other names as Ultracapacitor (UC) or Electrochemical Capacitor or Electric Double Layer Capacitor (EDLC). Supercapacitor offers high capacitance which means more energy storage and release high power that utilizes high surface area electrode materials and very thin electrolytic dielectrics that allows 
achieving capacitances several orders of magnitude larger than conventional capacitors [1-5]. In most of the application where constant energy is required, battery can supply but when repeated peak load is supplied because of high power demand at burst, the battery damages easily. While Supercapacitors can easily supply the repeated peak load and high power demand can be easily delivered to the load at burst.

Energy in a battery is normally stored chemically as active material in conducting electrode and releases electrical energy to the load terminals by making use of an oxidation/reduction reaction which transfers energy electrochemically through electrolyte. Because of this chemical reaction, its charging/discharging is slow process which makes a battery charging time high.

Supercapacitor on the other hand, works on the same principle of conventional capacitor where electrostatic charge is accumulated on the conductors while in SC; charges are accumulated at interface between the surface of a conductor and an electrolytic solution. This storage of energy or conversion of energy process does not involve redox reaction. Thus the internal resistance of the SC which controls the charging and discharging of SC is low. This internal resistance effect of all the connection circuitry is called Equivalent Series Resistance (ESR) which is another property to deliver high power burst. Supercapacitor stores energy in the same manner as capacitor but has very high capacitance as defined by the following relations.

$$
C=\frac{Q}{V} ; \quad C_{S C}=\varepsilon \frac{A}{D}=\varepsilon_{o} \varepsilon_{r} \frac{A}{D} \quad \& \quad E=\frac{1}{2} C_{S C} V^{2}
$$

Where $\mathrm{C}$ is capacitance; $\mathrm{Q}$ is charge; $\mathrm{V}$ is voltage; $\varepsilon$ is permittivity; $\mathrm{A}$ is area; $\mathrm{D}$ is distance and $\mathrm{E}$ is energy.

Double layer thickness of the Supercapacitor depends on concentration of the electrolytic solution and on the size of the ions which is in the order of 5-10 $\AA$ for concentrated electrolytes. The capacitance of the double layer is about $10-20 \mu \mathrm{F} / \mathrm{cm}^{2}$ for a smooth electrode in concentrated electrolytic solution with the assumption of a relative dielectric constant $\varepsilon_{r}$ of 10 for water in the double layer [4]. Schematic diagram of Supercapacitor is presented below in figure 2 .

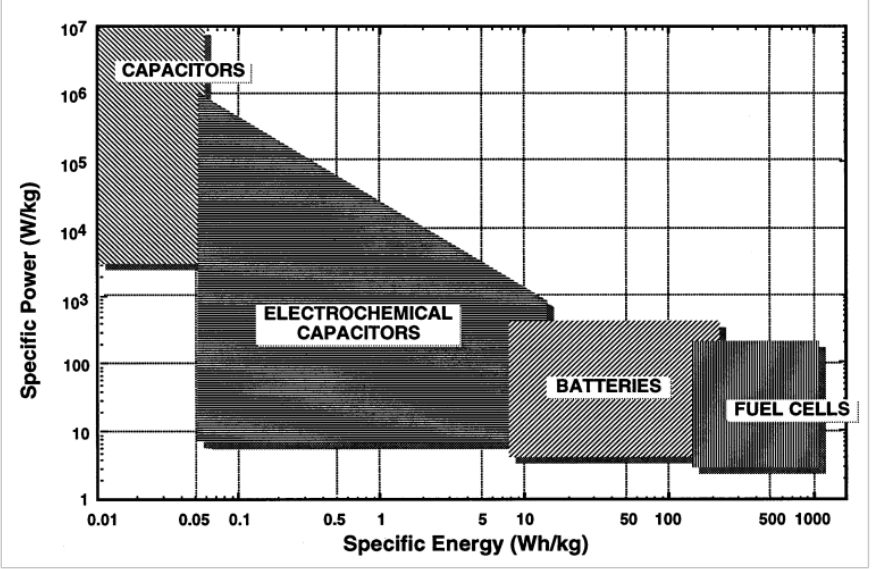

Figure 1:- Ragone Plot of Energy Storage Devices

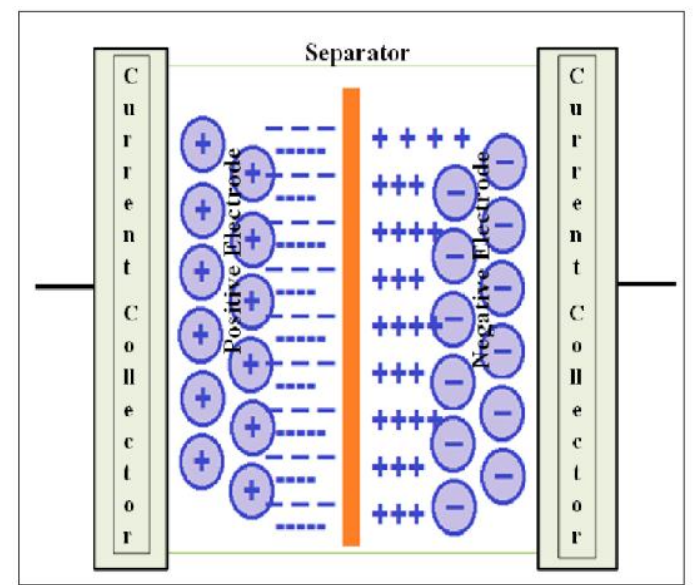

Figure 2:- Schematic diagram of Supercapacitor

\section{Classification of Electrochemical Supercapacitor:-}

Supercapacitors are distinguished by different criteria based on type of electrode material used, the type of electrolyte or the design of a cell. Depending on the type of material utilized in making the electrode of Supercapacitor, there are three main types of Supercapacitor: carbon electrode, polymeric and metal oxide materials. Based on the possible materials suitable for making Supercapacitor is presented in the review [5]. The materials of both the electrodes are made of carbon materials then the charge formation in Supercapacitor is electrostatic (Helmholtz layer) and when any of the electrode or both uses metal oxide or conducting polymers then charge storage is faradaic [1].

\section{Electrode:-}

The electrode of the Supercapacitor can be of carbon materials such as Activated Carbons, Carbon Aerogels, Carbon Nanotubes or Carbon Fibers in EDLCs. In case of pseudocapacitors, Conducting Polymers or Metal Oxides are used 
along with or without carbon electrodes. The classification of Supercapacitor based on the types of materials used in preparation electrode is shown in figure 3.

- Carbons are available having a surface area (specific) up to $2500 \mathrm{~m}^{2} / \mathrm{g}$ as powders, woven cloths or fibres [2].

- A specific capacitance of very high value of up to $750 \mathrm{~F} / \mathrm{g}$ has been reported earlier for $\mathrm{RuO}_{2}$ prepared at relatively low temperatures [6].

- When conducting polymers are used as electrodes and compared to metallic oxides, which exhibit a series of redox potentials [7].

- Graphene based electrode for Supercapacitor on scalable production of graphene oxides (GOs) by a chemical treatment in the run of improving the electrical and mechanical properties there by increasing efficiency of energy storage.

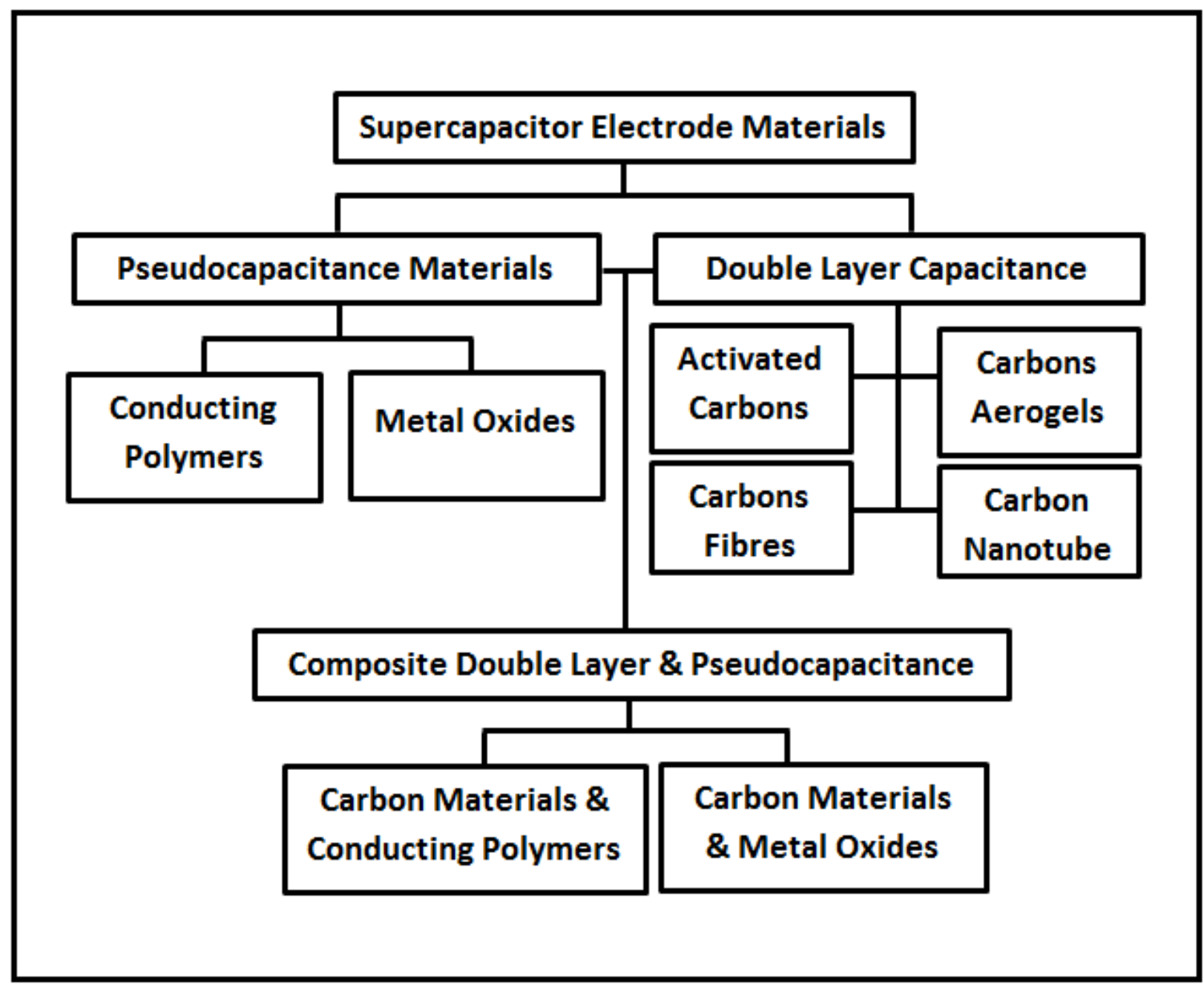

Figure 3: Classification of Supercapacitors based on Electrode Material.

\section{Electrolyte:-}

Supercapacitor can also be classified based on the type of electrolyte used. There are basically two types of electrolytes used in SC: Aqueous/Non-Aqueous and Organic/Inorganic. The greater the contents of ions in electrolyte higher are the conductivity. Electrolyte plays a major role in deciding the performance, safety and lifetime of SC. It is the physical and electrochemical property of electrolyte that also determines the value of ESR and power ratings.

\section{Organic/Inorganic:-}

The available capacitors uses organic electrolyte as it can achieve high per unit cell voltage which may be above 2 $\mathrm{V}$. The cell float voltage of SC is typically $2.3 \mathrm{~V}$ that may go up to $2.7 \mathrm{~V}$ for a short time. This limit to increment is possibly because of the water content of the electrolyte. To further increase, some companies go for float voltage up to $3.2 \mathrm{~V}$ where extreme purification procedures of special electrolyte have to be applied [8]. Inorganic aqueous 
electrolytes are basically sulphuric acid, potassium hydroxide, ammonium salt dissolved in propylene carbonate. Organic electrolytes are of Tetraethylammonium Tetrafluoroborate, ester based solvent Propylenecarbonate, Acetonitrile [1].

\section{Aqueous/Non-Aqueous:-}

Aqueous electrolytes limits the cell voltage of electrochemical capacitors to typically $1 \mathrm{~V}$, but there is advantage which provides higher conductance and less rigid during purification and drying. They have high ionic conductivity with electrochemical operating window about $1.2 \mathrm{~V}$ of which it is neither reduced nor oxidized [3]. Water solvent when treated with alkalis such as potassium hydroxide, acids such as sulfuric acid, or salts such as sodium perchlorate, quaternary phosphonium salts, lithium perchlorate or lithium hexafluoride arsenate, water gives relatively high conductivity values around 100 to $1000 \mathrm{mS} / \mathrm{cm}$. Non-Aqueous electrolyte is preferred because of higher operating voltage range thus higher energy \& power densities.

\section{Modeling of Supercapacitors:- \\ Model of Supercapacitor:-}

An energy storage device Supercapacitor which has the working principle similar to conventional capacitor but with the formation of double capacitance and high capacity to store energy at the same time release high power as compared to capacitor.

The current flowing though the capacitor is governed by;

$$
I_{S C}=\frac{d Q}{d t}
$$

The charge stored in the capacitor is

$$
Q=C_{S C} V_{S C}=\int I_{S C} d t
$$

Where $C_{S C}$ is the capacitance of the Supercapacitor; $V_{S C}$ is the voltage across the Supercapacitor

So to find the capacitance of SC from the data available from the manufacturer, one can use the equation;

$C_{S C}=\frac{d Q}{d V_{S C}}$

There are many electrical models available for the Supercapacitor which the researchers have developed as per the application or as per their requirement of accuracy in the application. Some of the commonly used electrical equivalent models of Supercapacitor are listed down.

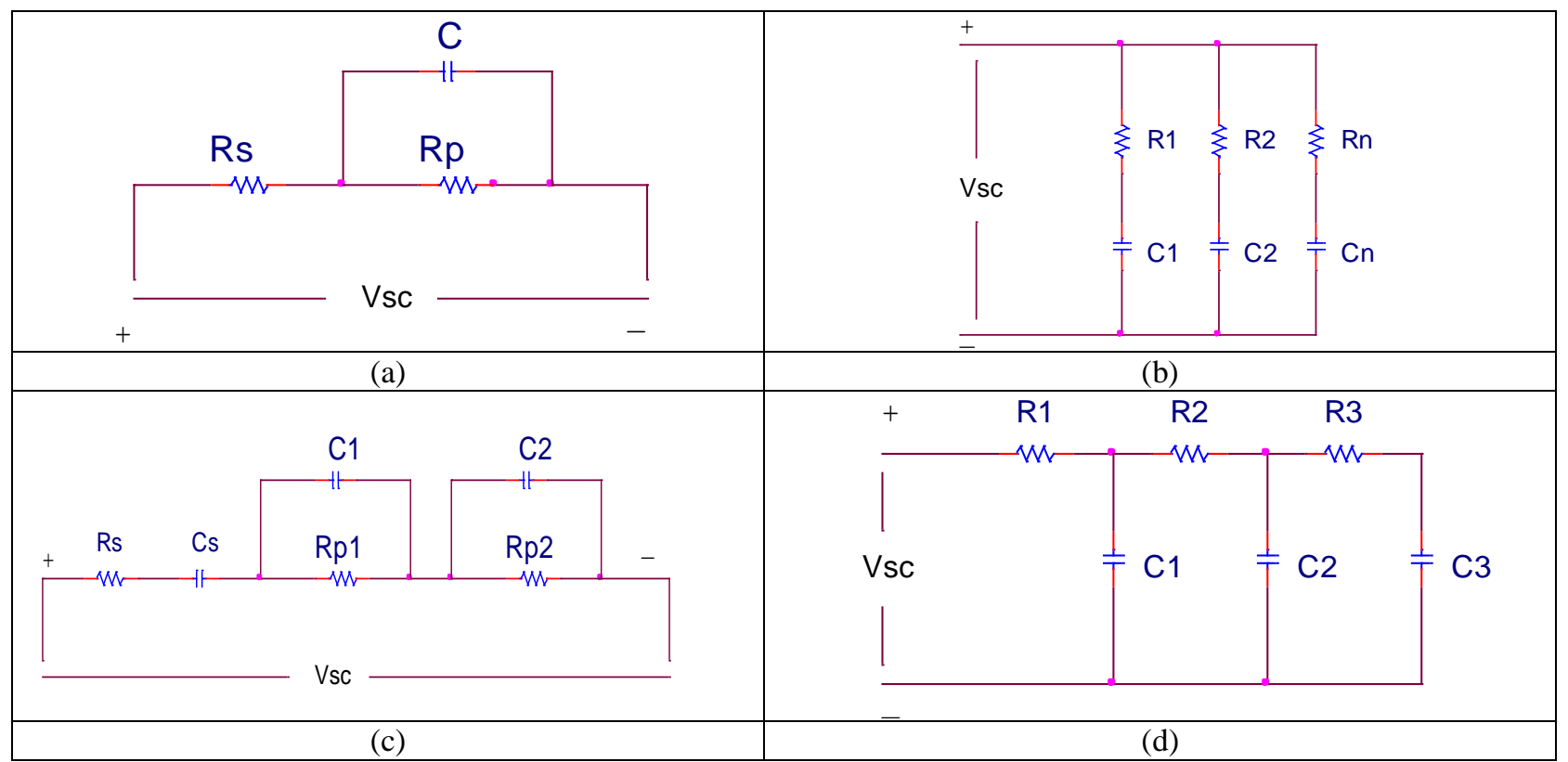




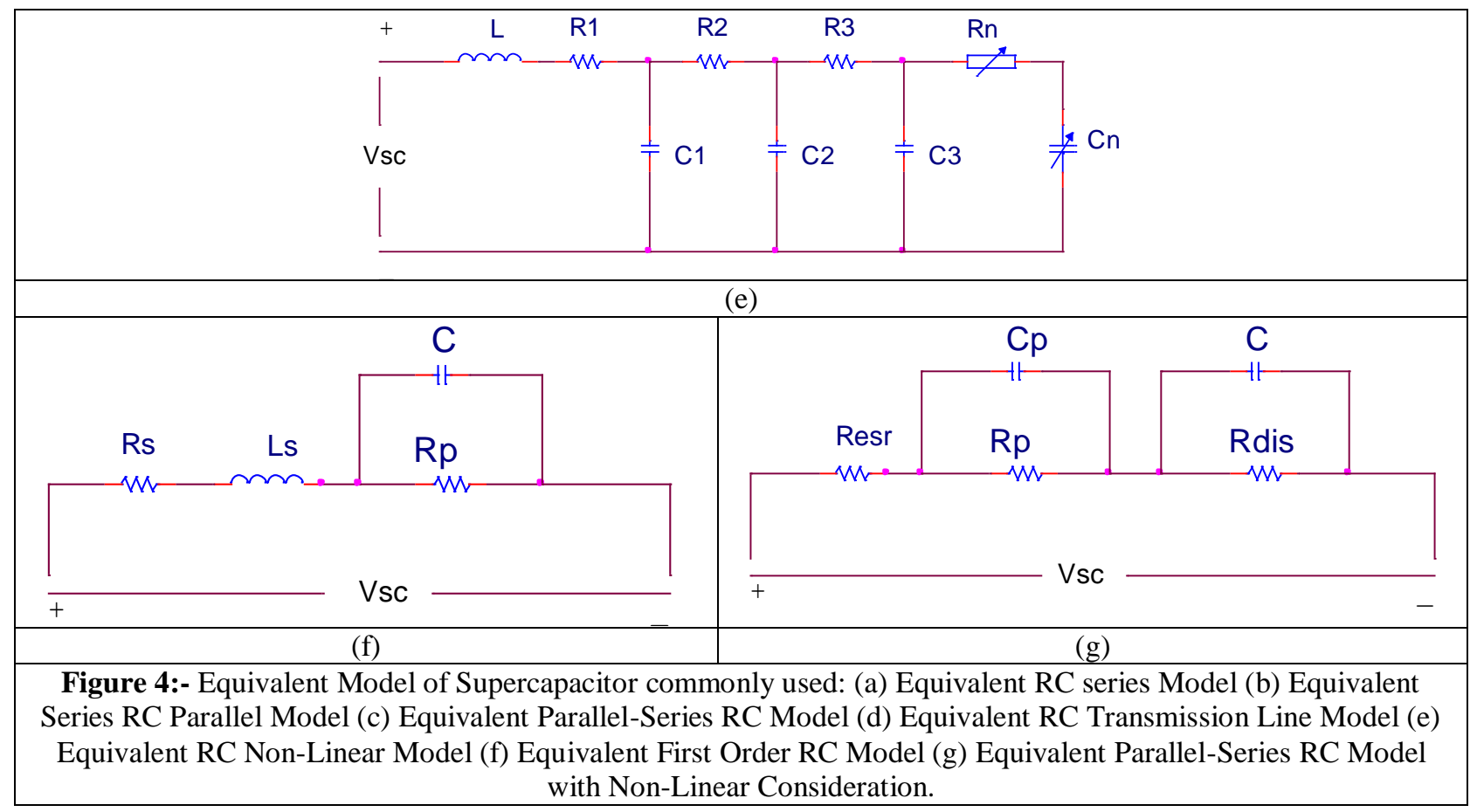

The equivalent circuit given in figure $4(\mathrm{~g})$ is model of a Supercapacitor manufactured by ECOND which is used in simulation to find the behavior. The measurement process to extract the details of SC is done by following certain procedure. First the SC is charged for certain period till the SC is fully charged and kept in this condition for some minutes to check the state of supercapacitor self-discharge. Now the supercapacitor is applied with negative source to allow forceful discharge to find the behavior during release of energy from supercapacitor when load is connected. Thus the values of the model are calculated using the relations:

\section{For calculating values of equivalent model in figure 4(g):-}

- The main capacitance of the SC which tells how charged is handled, amount of energy stored and the rate of energy variation level.

$$
C=\frac{Q}{V_{S C}}
$$

Where $\mathrm{C}$ is capacitance, $\mathrm{Q}$ is charge and $\mathrm{V}_{\mathrm{SC}}$ is the voltage level

- The resistance $\mathrm{R}_{\mathrm{ESR}}$ which is the equivalent series resistance of SC which accounts the charging and discharging voltage drop. This parameter decides the release of power from supercapacitor.

$R_{E S R}=\frac{d V}{d I}$

Where $\mathrm{V}$ is voltage drop, $\mathrm{I}$ is current, this small change in voltage and current can be found from the charging and discharging curve.

- The self-discharge behavior of the supercapacitor is represented by $\mathrm{R}_{\mathrm{DIS}}$ in the model cannot be easily found from the measurements data whose value has to be a large one as the self-discharge is generally very small.

- The capacitance connected in parallel $C_{P}$ value is to be taken as one thirteenth of $\mathrm{C}$, the main capacitance. This is the relation as suggested by the manufacturer considering the internal physical condition of the supercapacitor whose impact in the accuracy is very small and the resistance connected in parallel $R_{P}$ is again hard to find as it is not visible to the data measured from the curve so it is assumed with best matching from data where $\mathrm{C}_{P}$ and $\mathrm{R}_{\mathrm{P}}$ are included in the modeling to define the nonlinear behavior of the supercapacitor [9-10].

\section{Simulation of Supercapacitor Model:-}

The proposed model is simulated using the calculated values and choosing certain values as best suitable as shown in table 1 . 
Table 1:- Values of Supercapacitor Model

\begin{tabular}{|c|c|}
\hline Parameter & Value \\
\hline Nominal Voltage & $12 \mathrm{~V}$ \\
\hline Nominal Main capacitance, C & $300 \mathrm{~F}$ \\
\hline Equivalent Series Resistance, $\mathrm{R}_{\mathrm{ESR}}$ & $2 \mathrm{~m} \Omega$ \\
\hline
\end{tabular}

The supercapacitor is charged with 10 ampere for duration of 7 minutes till it is fully charged then kept for about 10 minutes to find the self-discharge behavior of SC. There after the SC is forcefully discharged with 20 ampere negative source to allow discharging for depth discharge analysis. The simulation result of charging and discharging as per desired durations to analyze the effect is given in figure 5. Along $\mathrm{X}$ axis is the time duration and along $\mathrm{Y}$ axis is the supercapacitor voltage and charging/discharging current. It is observed that after SC is fully charged, it drops for some voltage when the charging source is removed but is very low indicating that self-discharge is very low. At the end of force discharge interval about 2 minutes, SC has still some voltage to show the charge availability. To further investigate when the SC will fully discharge, the negative source application interval is increased to 4 minutes. Now, as is clearly shown in figure 6 , that the $\mathrm{SC}$ is completely discharged at about 3 minutes 23 seconds after the application of force discharge step.

To further investigate the behavior of the Supercapacitor model, the Equivalent Series Resistance is varied from a minimum of $0.1 \mathrm{~m} \Omega$ to $1 \Omega$ resistance in the model and checked the result as shown in figure 7 . It is evident from the result that for normal operation of ESR values around the calculated value, there are some charges left out in SC but as the ESR increases the discharging action increases during the same interval of forced discharge process. It is found that at $220 \mathrm{~m} \Omega$ of resistance the discharge is completed discharge state. There after the SC starts charging in opposite polarity that will eventually degrade the life of supercapacitor in long run.

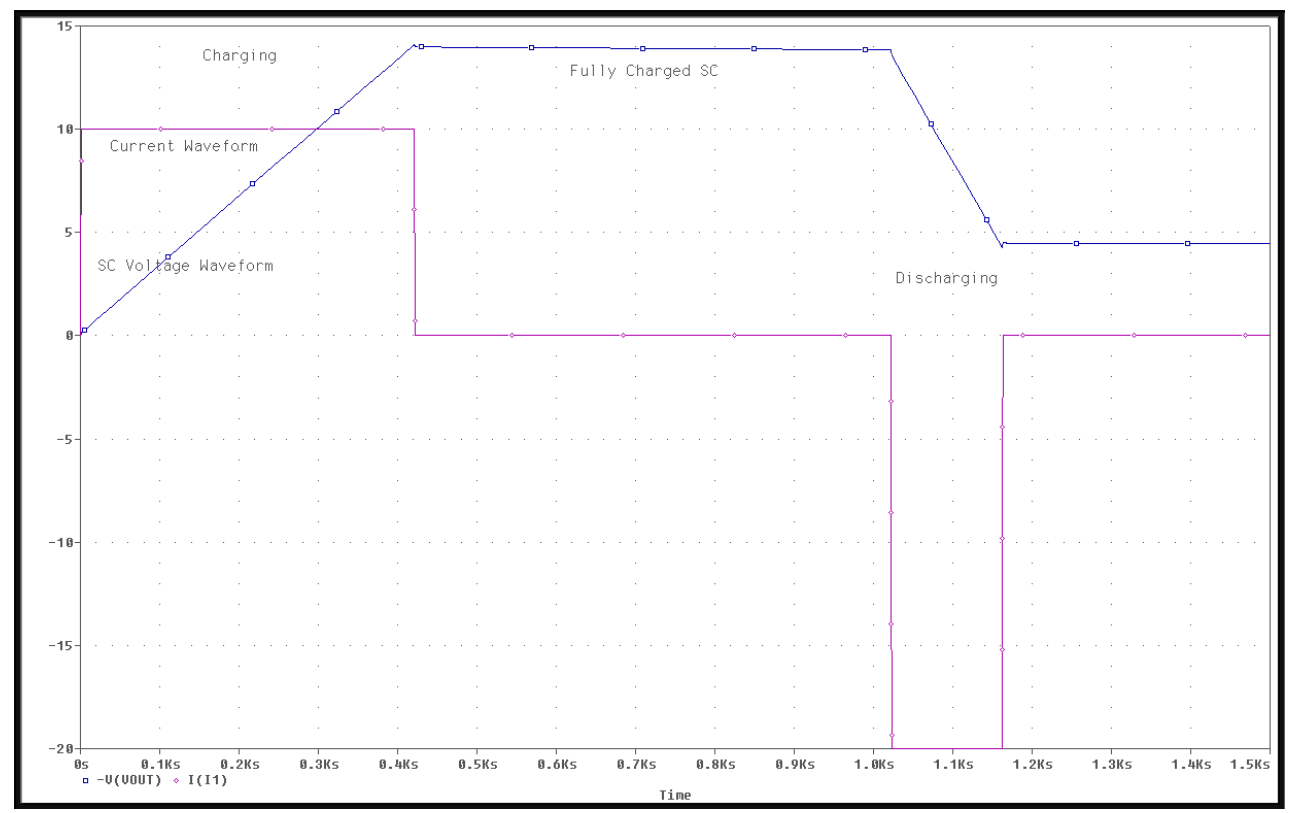

Figure 5:- Charging and Discharging of Supercapacitor. 


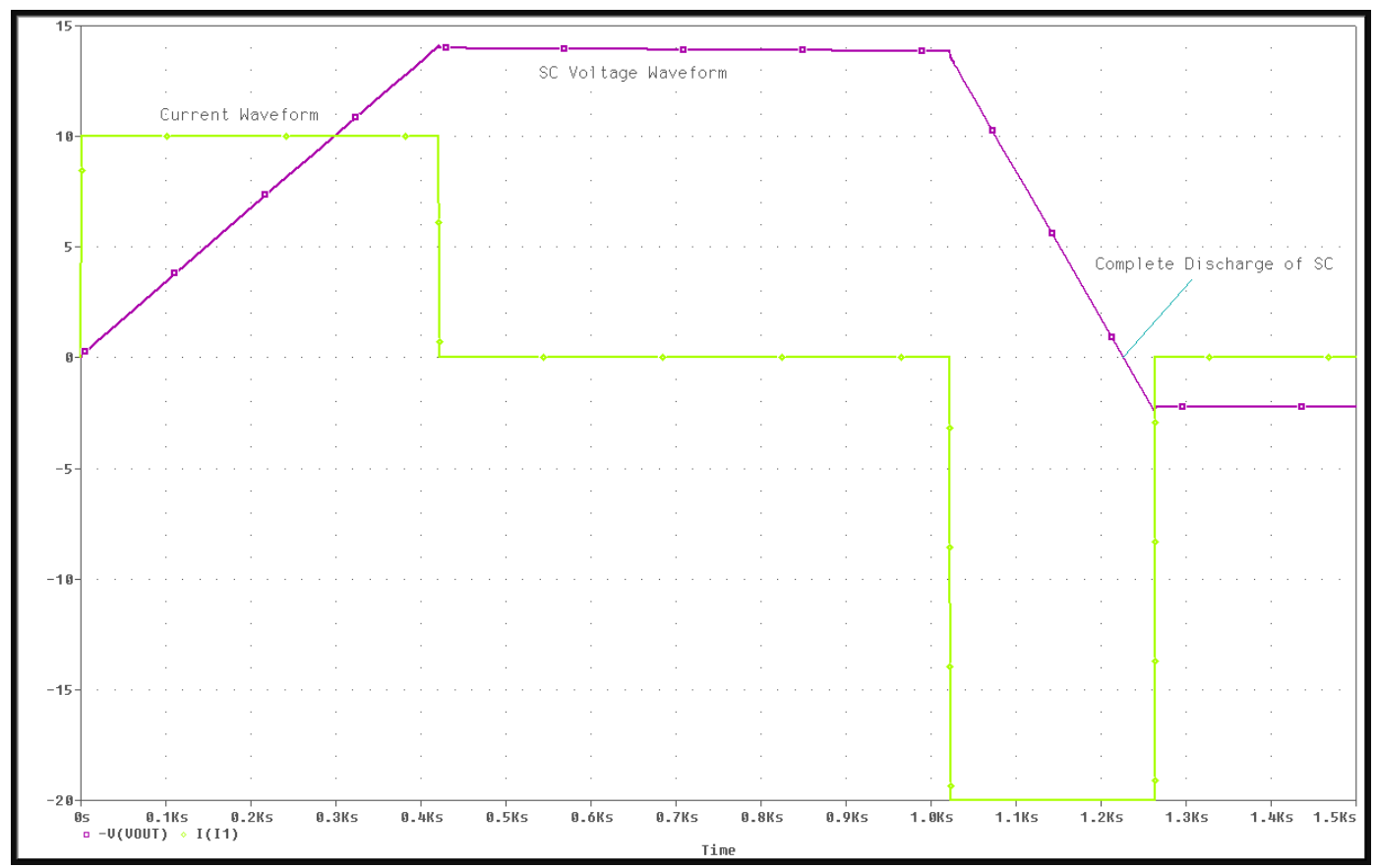

Figure 6:- Supercapacitor with complete discharge.

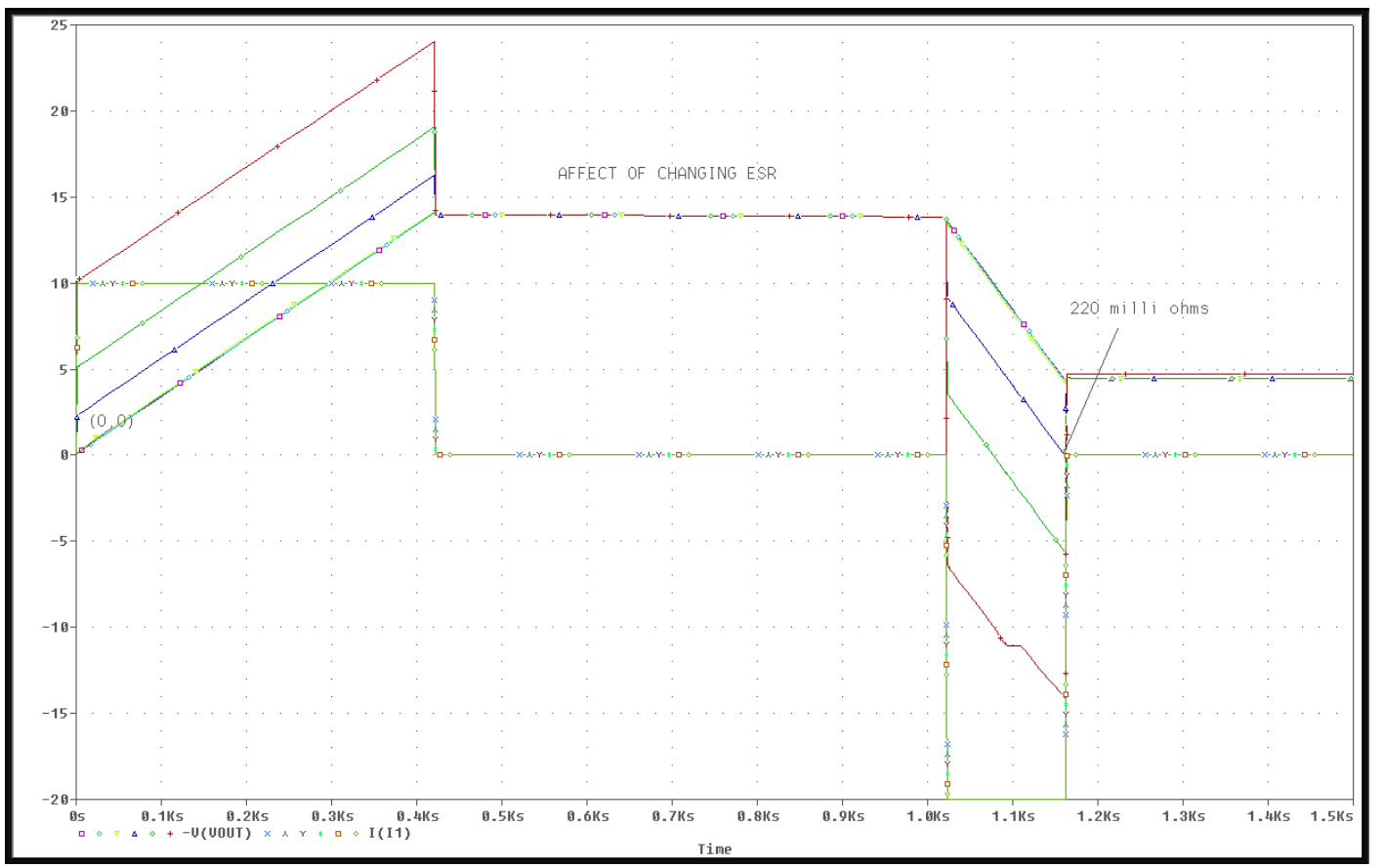

Figure 7: Supercapacitor for Different Values of ESR

\section{Conclusion:-}

Almost in all the applications, batteries were used as primary or secondary source of power. But with the advancement of new technology of energy storage, almost all the applications where battery was used can be replaced by Supercapacitor. The supercapacitor from the analysis found that there is some self-discharge taking place as indicated by the double breakdown during the discharge interval but of little significance. It continues to retain certain voltage level unless otherwise it is forcefully discharged. The high burst of power from the Supercapacitor is the effect of the value of the ESR. The variation of ESR result that higher the value of resistance lower the power burst that means higher the energy storage capacity. There is requirement of selection of best and 
proper electrolyte to maintain suitable ESR. Thus the supercapacitor can be used where the peak load demand is of higher priority and at the same time it can also store large amount of energy which facilitates many applications where high power and high energy is required that failed by batteries to deliver high peak load.

\section{References:-}

1. Conway, B. E. (1999), "Electrochemical Supercapacitors: Scientific Fundamentals and Technological Applications", New York, Kluwer-Plenum.

2. Burke, A. (2000), "Ultracapacitors: why, how, and where is the technology", Journal of Power Sources 91(1): $37-50$.

3. Kotz, R. and M. Carlen (2000), "Principles and applications of electrochemical capacitors", Electrochimica Acta 45(15-16): 2483-2498.

4. B.E. Conway, J. O’M. Bockris, I. A. Ammar, Trans. Faraday Soc. 47 (1951) 756.

5. S. Sarangapani, B.V. Tilak, C.P. Chen, J. Electrochem, Soc. 143, (1996), 3791.

6. J.P. Zheng, P.J. Cygan, T.R. Jow, J. Electrochem. Soc. 142 (1995) 2699.

7. K. Naoi, Extended Abstracts, $49^{\text {th }}$ Annual Meeting of the International Society of Electrochemistry, Kitakyushu, Japan, September, pp. 647, 1998.

8. A. Yoshida, K. Nishida, S. Nonaka, S. Nomoto, M. Ikeda, S. Ikuta, Japanese Patent 9-266143, (to Matsushita Electric), 1997.

9. P. Johansson, B. Andersson, "Comparison of Simulation Programs for Supercapacitor Modelling: Model Creation and Verification", Chalmers University of Technology, Sweden, 2008.

10. C. Farcas, D. Petreus, I. Ciocan, and N. Palaghita, "Modeling and simulation of supercapacitors", International Symposium for Design and Technology of Electronics Packages, pp. 195-200, 2009. 